\title{
Dermal flap of the lower pole of the breast for immediate reconstruction with alloplastic materials
}

\author{
Francisco Miguel Said-Lemus ${ }^{1 *}$, Daniel Garza Arriaga², Fernando Daniel Chávez-Osorio² and \\ José Daniel Guerra-Rendón ${ }^{3}$
}

${ }^{1}$ Plastic, esthetic an d reconstructive surgeon, Head of the Department of Reconstructive Surgery, Instituto de Enfermedades de la Mama, FUCAM, AC; ${ }^{2}$ Plastic, esthetic and reconstructive surgeon, Instituto de Enfermedades de la Mama, FUCAM (Fundación Cáncer de Mama); ${ }^{3}$ Plastic and reconstructive surgery resident, Hospital Central Sur de Alta Especialidad Pemex Picacho, Ciudad de México, Mexico

\begin{abstract}
Introduction: Immediate reconstruction has achieved better cosmetic results in addition to decrease surgical time for reconstruction. In those patients with a ptotic and large breasts (moderate to severe hypertrophy), candidates for skin-reducing mastectomy, a lower dermal flap can be made to cover the tissue expander and/or definitive implant, being a viable and affordable alternative to the use of acellular dermal matrix. Method: In the present study we present the experience of immediate breast reconstruction with a lower dermal flap in skin-reducing mastectomies in patients with breast ptosis II and III, performed in 2015 in the FUCAM, A.C. Hospital. Results: The use of the inferior dermal flap has been shown to have adequate aesthetic results with the security of providing adequate coverage to the alloplastic material used for reconstruction. Conclusions: It is a safe, reproducible and favorable technique in patients candidates for skin reduction mastectomy, offering an alternative to the dermal matrices.
\end{abstract}

Key words: Breast reconstruction. Subcutaneous mastectomies. Macromastia. Breast cancer. Tissue expander

Available online: 01-04-2019 Gac Mex Oncol. 2018;17:31-35 www.gamo-smeo.com 1665-9201 / @ 2017 Sociedad Mexicana de Oncología. Published by Permanyer México. This is an Open Access article under the terms of the CC BY-NC-ND license (http://creativecommons.org/licenses/by-nc-nd/4.0/) 


\section{Introduction}

Breast cancer remains one of the main malignant neoplasms in women, occuring in up to one out of every 8 women ${ }^{1}$. In $80 \%$ of cases it occurs sporadically, in $15 \%$, it is familial, and in $5 \%$, hereditary. There are various histological types, which will determine the prognosis and the treatment plan. Depending on patient conditions, histological type, disease progression and the treatment plan, immediate or late reconstruction will be able to be carried out ${ }^{2}$.

With technological and research advances, performing more conservative surgeries is currently sought, using more reconstructive techniques with better esthetic results. By 1963, the modern era of breast reconstruction was initiated with the introduction of breast implants. Tissue expanders started being used in 1972 with Radovan. By 1977, the use of latissimus dorsi extended flap was described, and in 1982, the use of transverse rectus abdominis myocutaneous (TRAM) flap². The main final goals of breast reconstruction include being able to produce volume, shape, projection and symmetry, as well as reconstruction of the areola-nipple complex and achieving an improvement in patients' physical appearance, improving quality of life, avoiding the use of external prosthesis and improving self-esteem, among others ${ }^{2,3}$. However, it remains a challenge, and with the advancement of years and technology, the results demand an improvement ${ }^{2}$. For each case, patients who are candidates to reconstruction for partial mastectomy should be assessed, as well as those who are to undergo total or risk-reducing mastectomies in order to establish the most adequate technique for them ${ }^{3}$. In addition, whether the patient will be candidate to any co-adjuvant treatment should be taken into account, among which the most important to consider because of the sequels is postoperative radiotherapy, and evaluate the performance of late or immediate-late reconstruction ${ }^{3}$.

Immediate reconstruction can be carried out in two forms: Using alloplastic materials (in one or two surgical times with expander and implants) and by means of autologous pediculated flaps or free flaps ${ }^{4}$. When reconstruction is performed with alloplastic material it is highly important for it to remain entirely covered, with local muscle flaps being used for this purpose. However, adequately covering the alloplastic material is not always possible, and the use of dermal matrices is therefore an important element in these situations.

One of the inconveniences of these dermal matrices lies both in cost and availability and, hence, the present work describes a covering technique using an inferior dermal flap for immediate breast reconstruction with tissue expander.

\section{Material and methodology}

The described technique was used in patients of the Institute of Breast Diseases of the Breast Cancer Foundation (FUCAM, A.C. - Fundación del Cáncer de Mama, A.C.), diagnosed with early stage breast carcinoma with grade II or III ptosis who were candidates to skin-reducing mastectomy, within the time period from January to December 2015. A total of 21 patients underwent reconstruction with this technique and all patients had a follow-up of at least 12 months after the initial surgical procedure.

\section{Surgical technique}

The steps to develop the surgical technique are next described. A skin-reducing mastectomy must be planned with markings performed using the Wise pattern (Fig. 1); subsequently, the inferior flap is de-epithelized to create the inferior dermal flap (Fig. 2). Standard mastectomy is then performed leaving the previously marked cutaneous flaps (Fig. 3). The major pectoralis muscle is then lifted from the inferior incision, the minor pectoralis muscle is laterally and partially lifted and serratus muscles are dissected (Fig. 4). Once the muscle pouch is performed, the tissue expander is introduced and covered with the muscles, with the closure of the pouch being made with absorbable suture to the extent possible (Fig. 5). Subsequently, the expander or definitive implant inferior pole is covered by bringing the dermal flap upper border together with the major pectoralis inferior border, with release of the flap lateral portion being possible in order to cover the muscle breach and the alloplastic material lateral portion that may have been left exposed with the inferior dermal flap, fixing it with absorbable suture, this way achieving a complete and safe covering of the prosthesis in order to reduce the risk of exposure (Fig. 6). Subsequently, a drain is placed and the cutaneous flaps are stitched with absorbable suture, with an inverted-T closure being left (Fig. 7).

\section{Discussion}

Breast cancer is one of the most common oncologic pathologies in our country. Post-mastectomy breast reconstruction is a widely performed procedure and is 


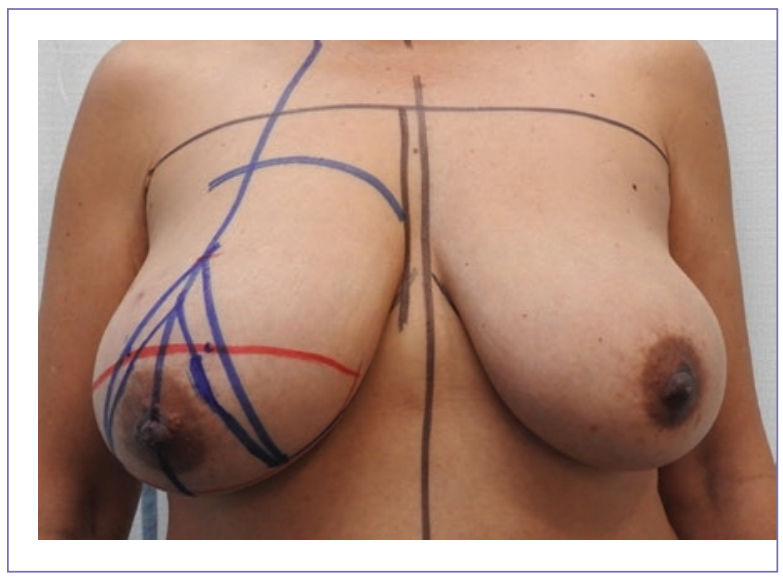

Figura 1. Preoperative markings with the Wise pattern.

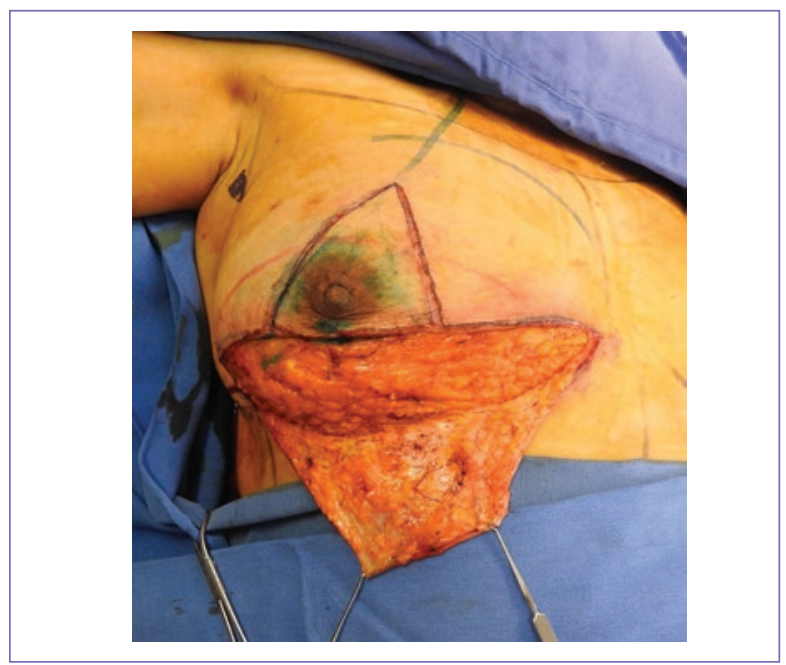

Figura 2. . Inferior dermal flap de-epithelization.

fundamental within reconstructive surgery. For breast reconstruction to be performed, there are different procedures, including reconstruction with autologous tissues (pediculated flaps or microsurgical flaps), use of alloplastic materials (expanders and implants) or a combination thereof (autologous flaps with the use of expanders and implants). In the case of breast reconstruction with alloplastic materials, in many cases the use of dermal matrices is necessary in order to favor their protection and avoid their exposure in case of complications.

Dermal matrix is an alternative for alloplastic material to be covered. The first dermal matrix product was Alloderm ${ }^{\circledR}$, obtained from human cadaveric matrix and available since 1994; the first report on its use was made by Breuing and Waring in 20055,6. After this,

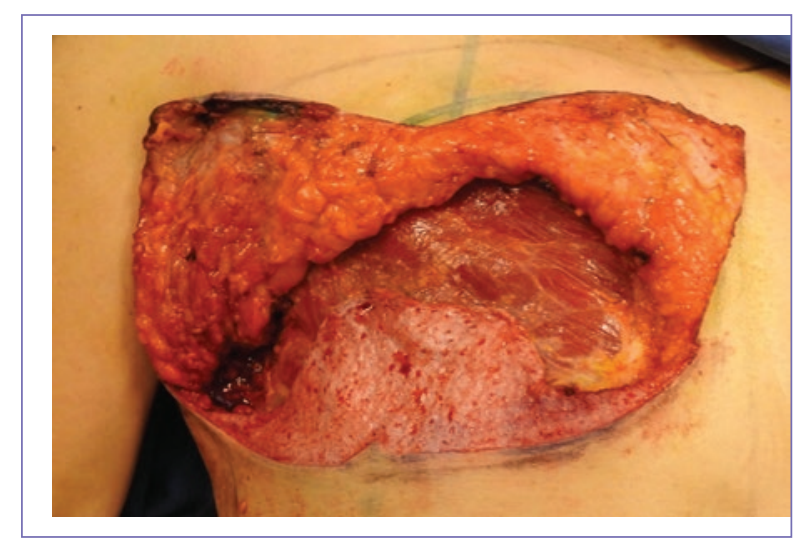

Figura 3. Mastectomy and dermal flap exposure.

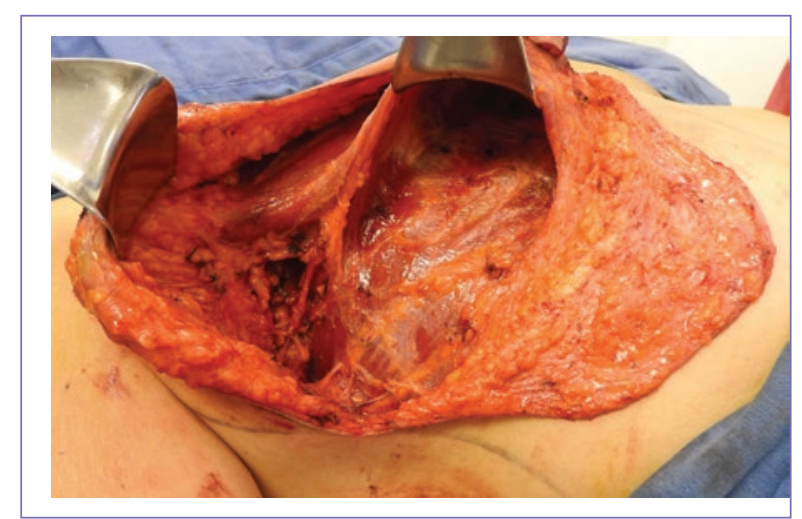

Figura 4. Muscle flaps lifting.

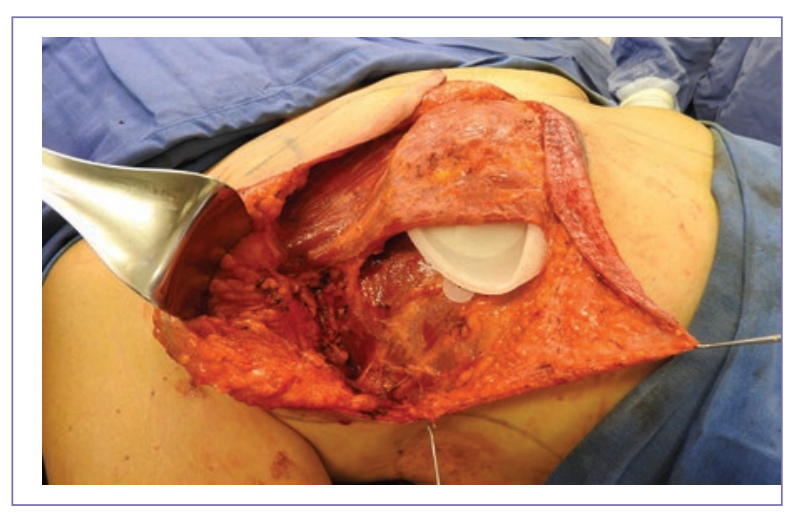

Figura 5. Expander introduction in the muscle pouch.

different dermal matrix products have been manufactured, some human-derived and others with porcine material. The use of biological matrices has gained popularity mainly in reconstructions with the use of expanders, since they provide cover and support 


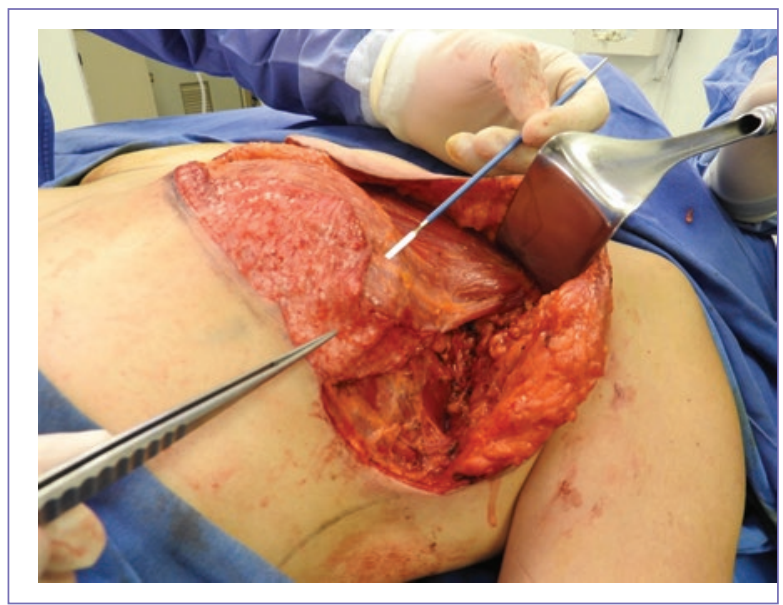

Figura 6. Expander covering with dermal flap.

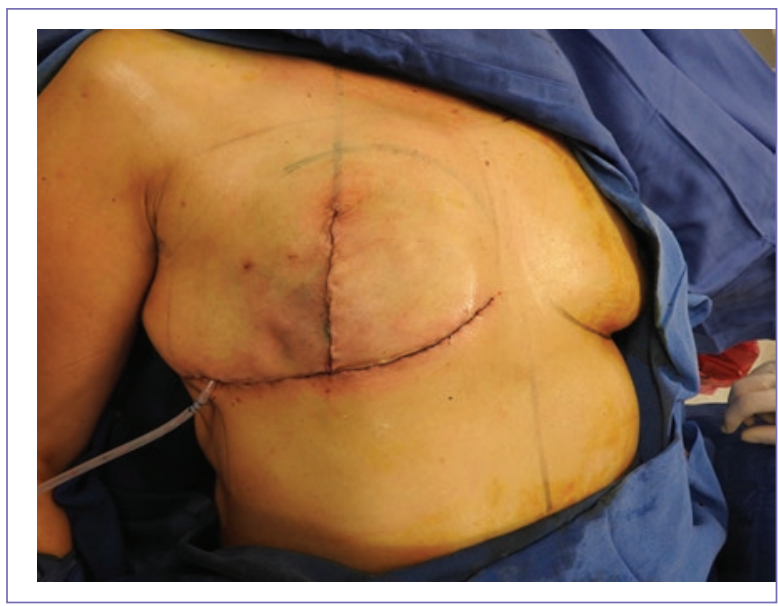

Figura 7. Cutaneous flaps closure with an inverted-T pattern

mainly to the breast inferior pole when adequate tissues (skin and/or muscle) are not available in order for alloplastic materials (expanders and implants) to be covered, it helps to define the inframammary ridge, it enables greater intraoperative expansion and, in addition, it reduces the tension at the moment of performing future expansions, it work as a support that favors vascularization for new tissue formation, and a lower rate of capsular contracture has even been reported in patients undergoing postsurgical radiotherapy ${ }^{7-16}$.

Complications that have been found with the use of dermal matrices include seromas, infection, wound dehiscence and matrix or alloplastic materials exposure. One of the main disadvantages of their use is the cost, but avoiding their use should be also considered in patients with cutaneous flaps inadequate vascularization, since this can cause their inadequate integration ${ }^{8}$.

There are cases where there are unfavorable conditions for reconstruction solely with alloplastic materials and where using a dermal matrix is not feasible. In these cases, breast reconstruction can be performed using an inferior dermal flap, which provides an adequate covering for the alloplastic materials that are to be used, either expanders or implants. There are reports on the use of dermal grafts as covering, which can be taken from the contralateral breast in case reduction simultaneous to mastectomy is required, or from the abdominal region ${ }^{17}$. In 1990, Bostwick described in skin-reduction mastectomies an immediate reconstruction in a single procedure placing a definitive implant and partially covering it with major pectoralis muscle and with a de-epithelized dermal flap. Subsequently, modifications to the technique have been made by Hammond, Losken, Nava and Ladizinsky, mainly using tissue expanders ${ }^{18}$. The main indication for the performance of an inferior dermal flap is in those patients with ptosis or macromastia who are to undergo a mastectomy and are candidates to skin-preserving mastectomy, and in whom the muscle pouch will not be sufficient to cover the alloplastic materials ${ }^{19-23}$ (Fig. 8). Complications that have been reported with this technique include seromas, hematoma and infection ${ }^{18}$.

The use of a dermal flap enables the covering of alloplastic material with the advantages that it doesn't increase the cost and that it can be used in patients with grade II or III mammary ptosis. In the cases of our Institution, of total operated patients, a minimum follow-up of 12 months was obtained with monthly assessment, where 4 patients experienced suffering of the cutaneous flap with partial necrosis evolution in one of them, and 3 patients experienced seroma. All cases were conservatively treated.

\section{Conclusions}

In patients with breast hypertrophy who are candidates to skin-reducing mastectomy, the post-mastectomy breast reconstruction technique using dermal flap is a viable and reproducible option when alloplastic materials need to be protected and dermal matrices are not available. It shows good results on the short and long term, with a low rate of complications that can be conservatively managed. 


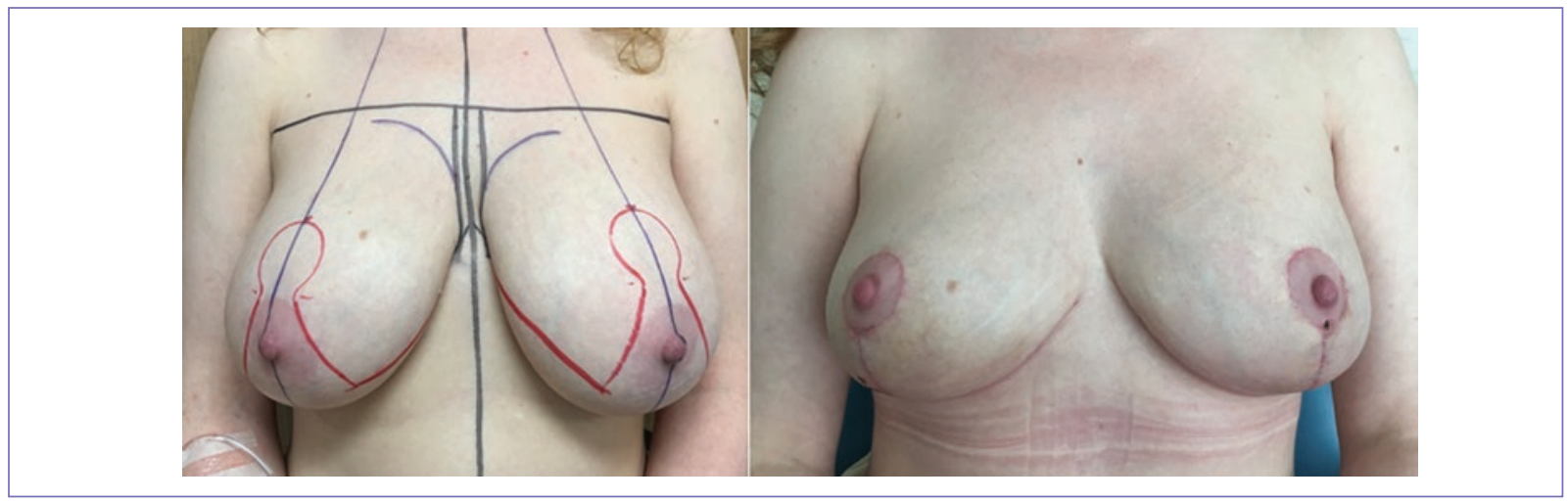

Figura 8. Dermal flap of the lower pole of the breast for immediate reconstruction with alloplastic materials

\section{Conflict of interests}

\section{The authors declare not having any conflicts of interests.}

\section{References}

1. Beahm E, Lang JE. Nelligan. Breast cancer: diagnosis, therapy and oncoplastic techniques. Vol. 5. Cap. 10. 265-295. Elsevier. 2013.

2. Hoover S, Kenkel J. Breast cancer, cancer prevention and breast reconstruction. SRPS. 2002;9(30).

3. Kronowitz S. State of the art and science in postmastectomy breast reconstruction. Plast Reconstr Surg. 2015;135:755e-71e.

4. Leff D, Bottle A, Gui G, et al. Trends in immediate postmastectomy breast reconstruction in the United Kingdom. Plast Reconstr Surg Glob Open. 2015;3:e507.

5. Haddad J, Rincón R, Hesiquio R. Reconstrucción mamaria inmediata mediante la utilización de matriz dérmica acelular o dermis autóloga. An Med (Mex). 2014:59(1):48-53.

6. Cheng A, Saint-Cyr M. Comparison of different ADM materials in breast surgery. Clin Plastic Surg.2012;39:167-75.

7. Bullocks J. DermACELL: a novel and biocompatible acellular dermalmatrix in tissue expander and implant-based breast reconstruction. Eur $\mathrm{J}$ Plast Surg. 2014:37:529-38.

8. Ibrahim A, Koolen P, Lin S, et al. Acellular dermal matrix in reconstructive breast surgery: Survey of current practice among plastic surgeons. Plast Reconstr Surg Glob Open. 2015;3:e381.

9. Jordan S, Khavanin N, Kim J, et al. An algorithmic approach for selective acellular dermal matrix use in immediate two-stage breast reconstruction: indications and outcomes. Plast Reconstr Surg. 2014;134:178-88.

10. Haddock N, Levine J. Breast reconstruction with implants, tissue expanders and alloderm: predicting volume and maximizing the skin envelope in skin sparing mastectomies. Breast J. 2010;16(1):14-9.
11. Chepla K, Dagget J, Soltanian H. The partial AlloDerm sling: Reducing allograft costs associated with breast reconstruction. J Plast Reconstr Aesthet Surg. 2012;65:924-30.

12. Topol B, Dalton E, Ponn T, et al. Immediate single-stage breast reconstruction using implants and human acellular dermal tissue matrix with adjustment of the lower pole of the breast to reduce unwanted lift. Ann Plast Surg. 2008;61:494-9.

13. Krishnan $\mathrm{N}$, Chatterjee $\mathrm{A}$, Van Vliet $\mathrm{M}$, et al. A Comparison of acellular dermal matrix to autologous dermal flaps in single-stage, implant-based immediate breast reconstruction: a cost-effectiveness analysis. Plast Reconstr Surg. 2013;131:953-61.

14. Frey J, Alperovich $\mathrm{M}$, Weichman $\mathrm{K}$, et al. Breast reconstruction using contour fenestrated alloderm: does improvement in design translate to improved outcomes? Plast Reconstr Surg Glob Open. 2015;3:e505.

15. Gdalevitch P, Ho A, Genoway K, et al. Direct-to-implant single-stage immediate breast reconstruction with acellular dermal matrix: predictors of failure. Plast Reconstr Surg. 2014;133:738e-747e.

16. Venturi M, Mesbahi A, Boehmler J, et al. Evaluating sterile human aceIlular dermal matrix in immediate expander-based breast reconstruction: a multicenter, prospective, cohort study. Plast Reconstr Surg. 2013; 131:9e-18e.

17. Hudson D, Adams K, Adams S. Autologous dermal graft in breast reconstruction. Ann Plast Surg. 2012;68:253-6.

18. Ladizinsky D, Sandholm $P$, Jewett $S$, et al. Breast reconstruction with the bostwick autoderm technique. Plast Reconstr Surg. 2013;132:261-70.

19. Torstenson T, Boughey J, Saint-Cyr M. Inferior dermal flap in immediate breast reconstruction. Ann Surg Oncol. 2013;20:3349.

20. Derderian Ch, Karp N, Choi M. Wise-pattern breast reconstruction. Modification using alloderm and a vascularized dermal-subcutaneous pedicle. Ann Plast Surg. 2009;62:528-32.

21. Carlson G, Bostwick J, Styblo T, et al. Skin-sparing mastectomy. Oncologic and reconstructive considerations. Ann Surg. 1997;225(5):570-5.

22. Nava M, Cortinovis $U$, Ottolenghi J, et al. Skin-reducing mastectomy. Plast Reconstr Surg. 2006;118(3):603-10.

23. Hammond D, Capraro Ph, Ozolins E, et al. Use of a skin-sparing reduction pattern to create a combination skin-muscle flap pocket in immediate breast reconstruction. Plast Reconstr Surg. 2002;110(1):206-11. 